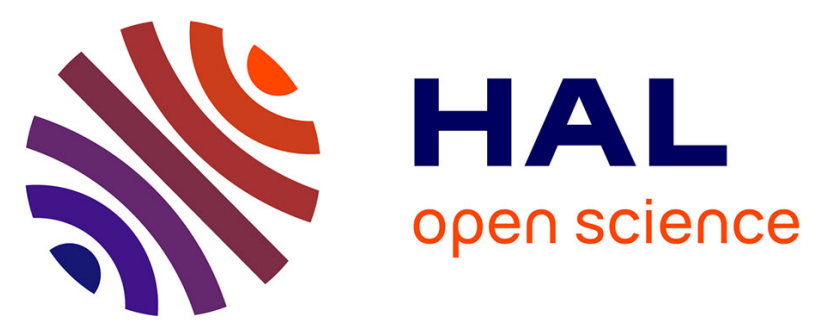

\title{
The role of microcrystalline structure on optical scattering characteristics of semi-crystalline thermoplastics and the accuracy of numerical input for IR-heating modeling
}

Sinan Boztepe, Rémi Gilblas, Olivier de Almeida, Christian Gerlach, Yannick Le Maoult, Fabrice Schmidt

\section{To cite this version:}

Sinan Boztepe, Rémi Gilblas, Olivier de Almeida, Christian Gerlach, Yannick Le Maoult, et al.. The role of microcrystalline structure on optical scattering characteristics of semi-crystalline thermoplastics and the accuracy of numerical input for IR-heating modeling. International Journal of Material Forming, 2018, 11 (5), pp.717-727. 10.1007/s12289-017-1386-z . hal-01869104

\section{HAL Id: hal-01869104 https://imt-mines-albi.hal.science/hal-01869104}

Submitted on 18 Mar 2019

HAL is a multi-disciplinary open access archive for the deposit and dissemination of scientific research documents, whether they are published or not. The documents may come from teaching and research institutions in France or abroad, or from public or private research centers.
L'archive ouverte pluridisciplinaire HAL, est destinée au dépôt et à la diffusion de documents scientifiques de niveau recherche, publiés ou non, émanant des établissements d'enseignement et de recherche français ou étrangers, des laboratoires publics ou privés. 


\title{
The role of microcrystalline structure on optical scattering characteristics of semi-crystalline thermoplastics and the accuracy of numerical input for IR-heating modeling
}

\author{
Sinan Boztepe ${ }^{1} \cdot$ Rémi Gilblas $^{1} \cdot$ Olivier de Almeida $^{1} \cdot$ Christian Gerlach $^{2}$. \\ Yannick Le Maoult ${ }^{1} \cdot$ Fabrice Schmidt ${ }^{1}$
}

\begin{abstract}
Infrared (IR) heating is widely used for thermoforming of thermoplastic polymers. The key benefit of radiation heating is that a significant amount of the radiative energy penetrates into the polymers thanks to their semi-transparency. For the case of heating unfilled semi-crystalline polymers, the relation between their microcrystalline structure and optical properties is the key to develop a predictive IR-heating model as microcrystalline structure introduces an optically heterogeneous medium. In this study, a relation between the microcrystalline structure of a polyethylene (PE) and its effect on the thermo-optical properties was experimentally analyzed considering a two-step analysis. At very first step, the relation was analyzed considering samples with identical thicknesses and different morphologies, characterized here in terms of degree of crystallinity $\left(\mathrm{X}_{\mathrm{c}}(\%)\right.$ ). Using Fourier Transform Infrared (FTIR) spectroscopy and integrating sphere, optical characteristics of the PE samples were analyzed in near-infrared (NIR) and middle-infrared (MIR) spectral ranges. The analyses showed that a slight variation in $\mathrm{X}_{\mathrm{c}}(\%)$ has a great effect on the optical characteristics of $\mathrm{PE}$, particularly the transmission characteristics in NIR range. The wavelength-dependent effect of $\mathrm{X}_{\mathrm{c}}(\%)$ on the transmission behaviors opened a discussion about the fact that the microcrystalline structures -in particular spherulites or their substructures such as lamellae- are responsible for optical scattering. Using the optical properties obtained from the
\end{abstract}

Sinan Boztepe

sboztepe@mines-albi.fr

Institut Clément Ader (ICA), Université de Toulouse, CNRS, IMT Mines Albi, INSA, ISAE-SUPAERO, UPS, Campus Jarlard, 81013 Albi CT Cedex 09, France, Albi, France

2 Procter \& Gamble Services NV, Brussels Innovation Center, Temselaan 100, 1853 Strombeek Bever, Belgium two-step experimental analyses, two different thermo-optical properties were calculated, namely extinction and absorption coefficients, and used as a numerical input for the parametric numerical studies. The numerical studies were performed using an in-house developed radiation heat transfer algorithm RAYHEAT-. Both the experimental and numerical analyses demonstrated the importance of the optical scattering regarding the identification of thermo-optical properties, used as a numerical input for radiation heat transfer models.

Keywords Infrared heating $\cdot$ Semi-crystalline thermoplastics · Thermo-optical properties · Optical scattering $\cdot$ Crystallinity $\cdot$ Radiation heat transfer modeling

\section{Introduction}

The major advantage of IR radiation heating is that a portion of the radiative energy penetrates directly into the bulk of thermoplastic polymer because of its semi-transparency where high heat flux densities can be used to decrease the heating time without causing thermal damage to the polymer surface [1]. Polymer structures used in thermoforming processes are heated prior to forming stage. The temperature profile at the end of the heating stage has therefore a great effect on viscoelastic properties and thus on the mechanical behavior of polymers during forming. As a consequence, it is crucially important to have an extensive knowledge on the temperature distributions throughout the bulk volume of a polymer.

A numerical radiative heat transfer model may be helpful to predict temperature distributions and to optimize the heating conditions such as reducing the heating time and the input of energy. However, physical background of the radiation heat transfer into the bulk polymer needs to be understood adequately in order to develop a predictive model. In the last 
decade, several authors developed such radiative thermal models for various thermoplastic polymers at which the amount of absorbed radiation [2] or temperature fields [3-7] on the studied thermoplastics were predicted closely. The radiation heat transfer models used in those studies adopted the Beer-Lambert law, assuming an optically homogenous and non-scattering medium for bulk material so that the radiation attenuation through a medium was only the function of absorption. For amorphous or semi-crystalline thermoplastics with a low $\mathrm{X}_{\mathrm{c}}(\%)$, where the crystalline content in such polymers is much lower than their amorphous content, the optical scattering may be negligible. However, it may be an illdefined assumption considering the radiation propagation inside of an optically heterogeneous medium. Basically, the term -optically heterogeneous medium- represents the discontinuities existing inside of a heated medium that causes to change the direction of propagated radiation, named optical scattering. These discontinuities -also called scatterers- may be fiber-matrix interface in thermoplastic composites [8, 9], filled particles $[10,11]$ or microcrystalline structure of semicrystalline polymers $[10,12]$. Considering optically heterogeneity of a medium, some numerical models were proposed for simulating laser transmission welding (LTW) of filled thermoplastics $[11,13]$ and laser-assisted tape placement (ATP) processes of thermoplastic composites [14]. In those studies, the relation between the scatterers (filled particles or fiber) and the scattered radiation was established. Such a relation between optical scattering and microcrystalline structure is required to be established for building an accurate radiation heat transfer for unreinforced or unfilled semi-crystalline thermoplastics. Although some effort given to establish a relation between microcrystalline structure of such type of polymers and its optical scattering effect, those proposed relations were limited to $\mathrm{X}_{\mathrm{c}}(\%)$ where no detailed explanation was introduced for scatterers that may be related to the morphological characteristics $[15,16]$. Lebaudy et al. [15] developed a radiation heat transfer model for poly(ethylene terephthalate) (PET) considering its cold crystallization behavior under heating. Their model was based on both scattering and absorption characteristics of the studied material where the change in the direction of radiation propagation caused by optical scattering was taken into account. In their study, it was concluded that $\mathrm{X}_{\mathrm{c}}(\%)$ was the main factor that causes optical scattering during coldcrystallization of PET. Those analyses were extended by Denis et al. [16] where the optical scattering characteristics of the PET polymers with different degrees of crystallinity were experimentally analyzed. Based on the analyses, an empirical law was established between the $X_{c}(\%)$ and the scattering coefficient of PET. Although the experimental analyses in [16] also revealed that the scattering characteristics of the material with the same $X_{c}(\%)$ changes dramatically in the spectral range of $0.4-1 \mu \mathrm{m}$, no explanation was given why the optical scattering behavior changes under varying wavelength and at a constant crystallinity. A similar wavelength-dependent optical scattering relation was observed in $[17,18]$ where it was proposed that the microcrystalline structure, particularly spherulites or their substructures such as lamellae, are responsible for the optical scattering.

In our study, the effect of the microcrystalline structure on thermo-optical properties of PE was studied. As a first step, the PE samples were prepared with identical thickness and cooled down under two different cooling conditions so that two groups of samples with different morphologies were introduced and characterized in terms of different degrees of crystallinity obtained via Differential Scanning Calorimetry (DSC). The effect of microcrystalline structure on the thermo-optical characteristics of PE was initially analyzed comparing the bi-directional transmittance and reflectance of the samples obtained via FT-IR spectroscopy and integrating sphere respectively. The analyses revealed a strong coupling between crystallinity and the thermo-optical properties of PE, especially in the range of NIR. The analyses were extended adopting a comparative experimental analysis on the transmission characteristics of the polymer. Bi-directional and directional-hemispherical transmittances of the identical samples were determined and the amount of the scattered radiative flux was estimated. These measurements also allowed estimating the extinction and absorption characteristics of the polymer that were calculated based on a well-known relation in radiation heat transfer theory. At last, parametric numerical studies were performed using the calculated extinction and absorption characteristics of the polymer where also the boundary conditions and the thermal-physical properties of the polymer were kept identical. The numerical studies were done employing a preliminary radiation heat transfer model. The computations were performed using in-house developed radiation heat transfer algorithm -RAYHEAT- which is built based on ray-tracing method and Beer-Lambert Law. Thanks to the numerical analyses, the effect of optical scattering, as a numerical input, was analyzed regarding the predicted temperature profiles. The combined experimental and numerical analyses demonstrated the importance of the optical scattering considering the identification of thermo-optical properties, accuracy of the numerical inputs and their effects on the temperature profile predictions.

\section{Experimental procedure}

\section{Sample preparation}

The PE polymer in the form of pellets was injection molded and the plate-like PE samples with dimensions of $78 \times 78 \times 2.2 \mathrm{~mm}$ were fabricated. The main reason to fabricate samples via injection molding is to obtain void free samples as the void formations may affect the optical properties of 
polymers as reported in $[12,17]$. The injected plate-like samples were then cut into small pieces and compressed in order to obtain thin samples with controlled thickness. The compression procedure was started by heating the samples up to $180^{\circ} \mathrm{C}$ and holding at that temperature for $5 \mathrm{~min}$ for the purpose of melting the crystalline phase completely. The samples were then hold at that temperature for $5 \mathrm{~min}$ and compressed until the desired thickness. The samples were separated into three groups where the first and the second groups of samples were prepared with a thickness of $0.5 \mathrm{~mm}$ and cooled down under two different cooling conditions: free convection in still air (slow-cooled) and quenching (fast-cooled) in liquid nitrogen $\left(\mathrm{LN}_{2}\right)$. Thanks to this step, the first and second groups of samples were prepared with the identical material and thickness but different crystalline morphologies and proportions due to the two different cooling. The relation between the microcrystalline structure of PE and its optical characteristics were analyzed adopting two-step analysis. Initial analyses were performed using the first and second groups of samples where the analyses were extended using the third group. The third group of samples was prepared with thicknesses of $0.42,0.90$ and $1.82 \mathrm{~mm}$. All the sample groups that were prepared for the experimental analyses are presented in Table 1.

\section{Crystallinity measurements}

The determination of the $\mathrm{X}_{\mathrm{c}}(\%)$ was carried out using a Perkin-Elmer DSC 8000. For the each DSC scan, a piece of material with a mass of $2.5 \mathrm{mg}$ was cut from the compressed samples. In order to determine the crystallinity that is induced by to the two different cooling steps, the melting endotherms were used for the calculations of $\mathrm{X}_{\mathrm{c}}(\%)$. The heating scan was performed at $20{ }^{\circ} \mathrm{C} / \mathrm{min}$ at a temperature range of $20{ }^{\circ} \mathrm{C}$ to $180{ }^{\circ} \mathrm{C}$. Apart from obtaining information on $\mathrm{X}_{\mathrm{c}}(\%)$, the melting temperature $\left(\mathrm{T}_{\mathrm{m}}\right)$ of the polymer were also determined thanks to the analyses.

$\mathrm{X}_{\mathrm{c}}(\%)$ was defined based on the ratio between the enthalpy of fusion of the sample $\left(\Delta \mathrm{H}_{\mathrm{m}}\right)$ and the theoretical enthalpy of fusion of $100 \%$ crystalline $\mathrm{PE}\left(\Delta \mathrm{H}_{\mathrm{m}}^{\mathrm{o}}\right)$, as shown in Eq. 1 . The enthalpy of fusion of each sample was calculated integrating the melting peak obtained from the DSC curves and, $\left(\Delta \mathrm{H}_{\mathrm{m}}^{\mathrm{o}}\right)$ of PE was chosen from literature [19]:

$X_{c}(\%)=\frac{\Delta H_{m}}{\Delta H_{m}^{0}}(\%)$

\section{Optical characterizations}

As aforementioned, two groups of samples with different crystallinity, but with identical material and thickness, were prepared. Thanks to this step, the difference in the optical characteristics of the samples could be related to the difference in their $\mathrm{X}_{\mathrm{c}}(\%)$.After the initial analyses were done using the first and second group of samples, the third group was used in order to analyze how strong scattering exists in the PE polymer adopting two different transmittance measurements.

The optical characteristics of the samples were determined in the range of NIR $(0.8-2 \mu \mathrm{m})$ and $\operatorname{MIR}(2-25 \mu \mathrm{m})$. Transmittance $\left(\mathrm{T}_{\lambda}\right)$ of the PE samples was determined employing FT-IR spectrometer (Bruker Vertex 70) where also their reflectance $\left(\mathrm{R}_{\lambda}\right)$ was determined via mounting the integrating sphere as an external device on the spectrometer. The experimental setups for the transmittance and reflectance measurements are displayed in Fig. 1(a) and (b) respectively. The physical meanings of $T_{\lambda}$ and $R_{\lambda}$ are the ratio of the total amount of transmitted $\left(\Phi_{\lambda}{ }^{t}\right)$ and reflected $\left(\Phi_{\lambda}{ }^{r}\right)$ radiative flux over incident flux $\left(\Phi_{\lambda}{ }^{\mathrm{i}}\right)$ [20], as also shown Fig. 1. The incident flux $\left(\Phi_{\lambda}{ }^{i}\right)$ is the initial emitted photon flux sent by the spectrometer. In the case of testing an optically heterogeneous medium, the transmittance may only provide the directlytransmitted light, named as directional-directional transmission or "in-line transmission" in literature [21, 22]. Although Apetz el al. reported that forward-scattered photons in small angles may also be detected by the detector of spectrometer [21], this may be relatively small in comparison to the amount of directly-transmitted light and it is therefore neglected in this study. In addition, if multiple internal scattering is considered in an optically heterogeneous medium, it may then be required to take into account that the scattered light inside of the medium may come back into the direction of the incident light. In other words, the light may be scattered multiple times inside of the medium due to the optical heterogeneity and some amount of the scattered light may be back to the direction of the incident light and detected by the spectrometer. This phenomenon is known as radiation gain due to in-scattering in radiation theory [23]. However, determination of the multiple scattering phenomena may require an extensive experimental analysis which is not in the scope of the current study. Therefore the effect of multiple internal scattering was neglected for the transmission measurements. Based on these assumptions, the transmission measurements performed using the spectrometer lead to have the bi-directional
Table 1 The PE samples prepared for the calorimetric and optical analyses

\begin{tabular}{llll}
\hline & 1st group & 2nd group & 3rd group \\
\hline Cooling condition & Fast-cooled & Slow-cooled & Slow-cooled \\
Sample thickness $(\mathrm{mm})$ & 0.5 & 0.5 & $0.42,0.90$ and 1.82 \\
\hline
\end{tabular}


a

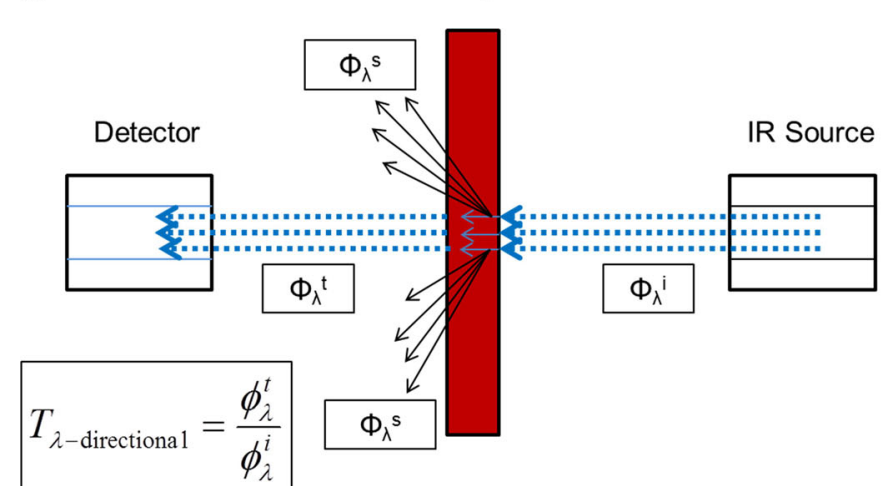

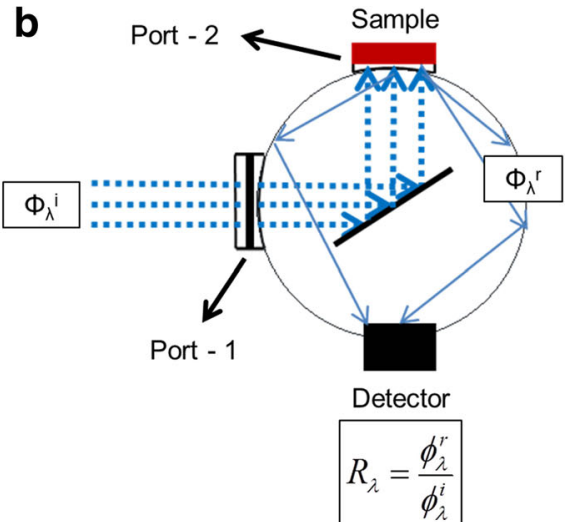

Fig. 1 Schematic representations of the experimental test setups of FT-IR spectrometer (a) and integrating sphere (b) for bi-directional transmittance and reflectance measurements respectively

transmittance of the samples where $\Phi_{\lambda}{ }^{\mathrm{t}}$ represents only the directly-transmitted light.

As displayed in Fig. 1(b), each sample was positioned at the side port of the integrating sphere (Port-2) in order to obtain $R_{\lambda}$. Apart from the measurement of $R_{\lambda}$, the integrating sphere was also used for the transmission measurements. For these tests, the samples were positioned at the entrance port (Port-1) of the integrating sphere so that directionalhemispherical transmittance of the samples was measured [24, 25] (Fig. 2). The key benefit of doing such measurement is to perform a comparative analysis considering the two different types of transmission measurements obtained from the identical samples. For the sake of simplicity, the bi-directional and the directional-hemispherical transmittance are now named as $\mathrm{T}_{\lambda \text {-directional }}$ and $\mathrm{T}_{\lambda \text {-hemispherical }}$ respectively. By assuming that the amount of the reflected light on the surface of an identical sample is the same for both the tests, the difference between the two transmission measurements is considered only due to the forward-scattered $\left(\Phi_{\lambda}{ }^{\mathrm{s}}\right)$ radiative flux. It is therefore assumed that radiative flux detected by the detector in the integrating sphere is the combination of both $\Phi_{\lambda}{ }^{\mathrm{s}}$ and

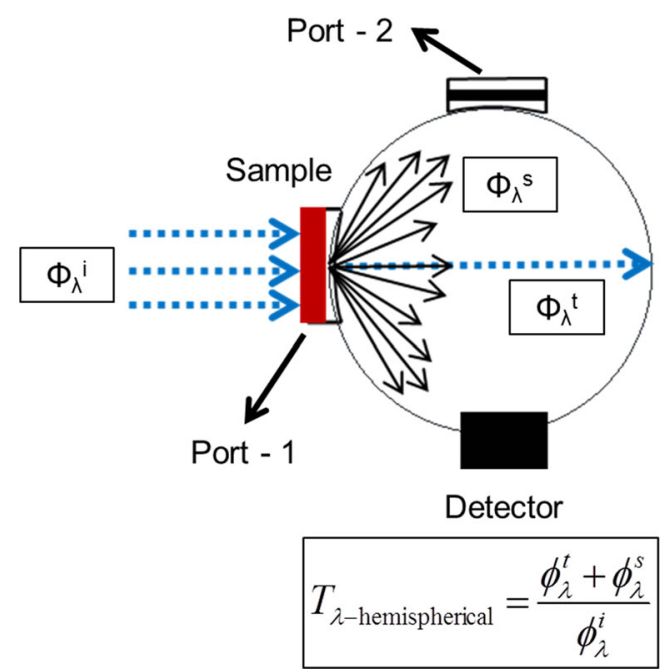

Fig. $2 \mathrm{~T}_{\lambda \text {-hemispherical }}$ measurements using the integrating sphere
$\Phi_{\lambda}{ }^{\mathrm{t}}$. It should also be noted that some amount of the radiative flux may also be back-scattered inside of the PE samples and may not enter the integrating sphere [25]. For the current case, the part of the back-scattered radiation was assumed negligibly small and only forward-scattering was considered. In our previous study [26], we analyzed how the $\mathrm{T}_{\lambda \text {-directional }}$ of PE increases dramatically under varying temperature where also $\mathrm{T}_{\lambda \text {-directional }}$ was compared to $\mathrm{T}_{\lambda \text {-hemispherical }}$ obtained at room temperature. One of the conclusion in that study was that the major part of the incoming radiation was forward-scattered, as the difference between the $\mathrm{T}_{\lambda \text {-directional }}$ measured at a temperature close to melting range and $T_{\lambda \text {-hemispherical was much }}$ smaller than the increase in $\mathrm{T}_{\lambda \text {-directional }}$ under heating. Similar analysis can be found in [27], where the ratio between incident power of laser light and transmitted and scattered light was measured for high density polyethylene (HDPE).

\section{Results and discussion}

\section{Experimental analyses between crystallinity and optical characteristics}

As explained in Section 2, the first and the second group of samples were prepared with the identical material and thickness but with different crystallinity induced by the two different cooling conditions. The actual cooling rate of the samples was not determined for both the cooling conditions as the focus of this study is to analyze the relation between optical properties of $\mathrm{PE}$ and its microcrystalline structure, represented here by different $\mathrm{X}_{\mathrm{c}}(\%)$.

The crystallinity of each sample was analyzed immediately after the compression and the cooling steps. Figure 3 illustrates the typical heating and cooling scans of the DSC analyses conducted in this study. In the figure, the two curves with upward and downward peaks represent the heating and cooling runs respectively. As it is seen in the figure, the upward peak - which is an endothermic reaction - provides 


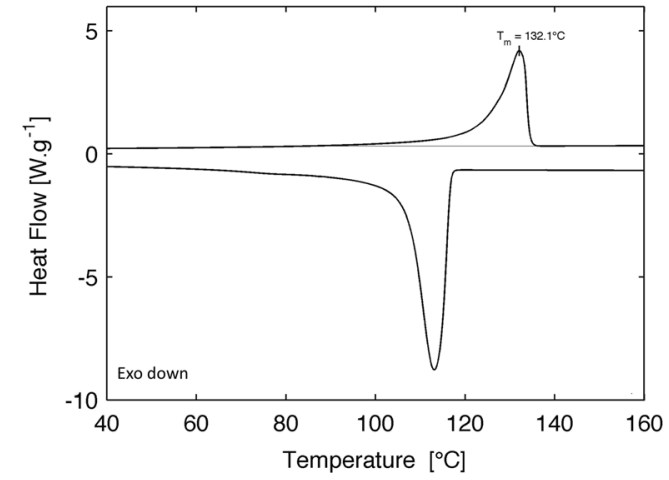

Fig. 3 Typical heating /cooling scan obtained during the DSC analyses of the PE samples

information on the melting point of the sample. The melting temperature $\left(\mathrm{T}_{\mathrm{m}}\right)$ of the sample was determined around $130{ }^{\circ} \mathrm{C}$. In addition, the melting enthalpy of each scan was calculated based on the integrated area under the melting peak, and the $\mathrm{X}_{\mathrm{c}}(\%)$ was determined. The analyses showed that the $X_{c}(\%)$ of the slow-cooled samples is $73 \%$ whereas the fastcooled samples have a $X_{c}(\%)$ with $67 \%$. It should be noted that the DSC measurements that were done for the identical samples in each group showed a $\mathrm{X}_{\mathrm{c}}(\%)$ that varies $+/-1 \%$ which is a conventional uncertainty of crystallization measurement obtained via calorimetric analyses. However, it is clear that the difference between the $\mathrm{X}_{\mathrm{c}}(\%)$ of the slowcooled and fast-cooled samples is significant which attributes the difference in the level of crystallinity and therefore different morphologies.

Transmission characteristics of the PE samples were initially analyzed measuring $\mathrm{T}_{\lambda \text {-directional }}$ of the fast and slow-cooled samples via FT-IR spectrometer. Figure 4 (a) and (b) illustrate $\mathrm{T}_{\lambda \text {-directional }}$ of the samples over the spectral ranges of 1-2 $\mu \mathrm{m}$ and 2-20 $\mu \mathrm{m}$, which are in the NIR and MIR regions respectively $[28,29]$. The $T_{\lambda \text {-directional }}$ measurements are clearly evident that a slight variation in the $\mathrm{X}_{\mathrm{c}}(\%)$ of PE has a great effect on its thermo-optical characteristics especially in a specific spectrum in NIR range. Conversely, the same trend is not seen in MIR range as the difference in crystallinity is somewhat effective on $\mathrm{T}_{\lambda \text {-directional }}$ until $3.5 \mu \mathrm{m}$ where after the measurements of both the slow and fast-cooled samples are very similar (Fig. 4 (b)). A similar trend was also observed in [17] where the transmission characteristics of semi-crystalline polypropylene (PP) studied and reported that the effect of optical scattering becomes ineffective with an increase in the wavelength of transmitted radiation especially in MIR range. It was also concluded in [17] that the crystalline lamellae plays a key role in optical scattering phenomenon in the PP polymer medium. The change in transmission behavior of the polymer is due to optical scattering phenomenon that is induced by different $\mathrm{X}_{\mathrm{c}}(\%)$ as only the directly-transmitted light is measured in $\mathrm{T}_{\lambda \text {-directional. }}$. However, this effect may not only be explained considering the difference in the degree of
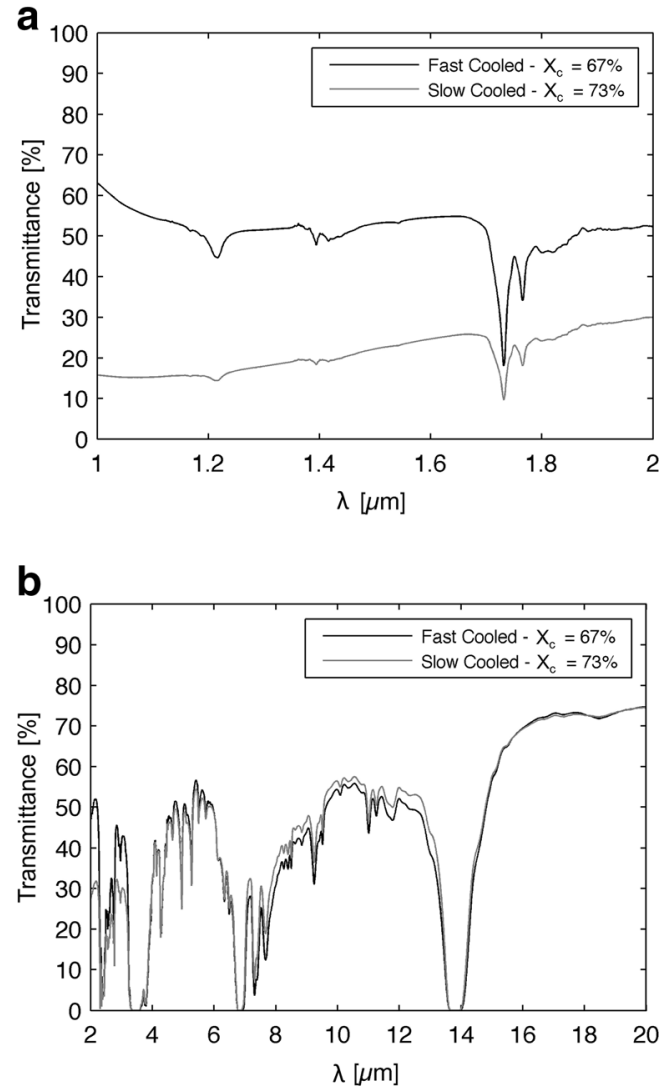

Fig. $4 \mathrm{~T}_{\lambda \text {-directional }}$ measurements in NIR (a) and MIR ranges (b) on the slow and fast-cooled samples with different $\mathrm{X}_{\mathrm{c}}(\%)$

crystallinities but also microcrystalline structure of the polymer as there is relation between the scattered light and the wavelength. More rigorously speaking, the relation between wavelength of radiation and the scatterer in the medium represented by spherulites or lamellae size as a microcrystalline substructure- is the key to govern the optical characteristics of unfilled semi-crystalline thermoplastics. The optical scattering behavior related to the spectral characteristics of a propagated radiation and morphological features (dimensions) of the scatterer in a semi-crystalline polymer can be explained thanks to the the well-known optical scattering theories such as Mie [11, 30], Rayleigh [31] and Rayleigh-Debye-Gans [21, $31]$ theories. In addition, considering that the set temperature of a typical IR heater is around $2400 \mathrm{~K}$ the maximum emission of the lamp $\left(\lambda_{\max }\right)$ is around $1.2 \mu \mathrm{m}$ regarding Wien's Displacement Law [23, 32]. Based on the Planck's curve for emitted spectral power, $96 \%$ of the emission of such an IRlamp occurs between $0.5 \lambda_{\max }$ and $5 \lambda_{\max }$. As it is seen in Fig. 4 (a), even an increase of $6 \%$ of the $X_{c}(\%)$ causes a decrease of nearly $50 \%$ of the $\mathrm{T}_{\lambda \text {-directional }}$ in the spectral range around $1 \mu \mathrm{m}$ that is close to the maximum emission wavelength of a typical IR heater and the difference is significant until $3.5 \mu \mathrm{m}$. Therefore the effect of the microcrystalline structure on the transmission characteristics in NIR and MIR ranges may not be neglected in case of building a radiation 
heat transfer model for IR heating assisted thermoforming processes of semi-crystalline thermoplastics.

Reflection characteristics of the slow and fast-cooled samples defined as $R_{\lambda}$ showed that there is a relatively weak relation between crystallinity and $R_{\lambda}$ in contrast to the $T_{\lambda \text {-directional }}$ measurements. As displayed in Fig. 5, a slightly higher $\mathrm{R}_{\lambda}$ was obtained in NIR range (a) for the slow-cooled samples which has the higher $\mathrm{X}_{\mathrm{c}}(\%)$ whereas no different was observed in MIR range (b). It was also observed that $\mathrm{R}_{\lambda}$ of both the samples show a decreasing trend with an increase in wavelength over NIR range. In this range, the difference between $R_{\lambda}$ of the two samples is found around $3 \%$.

As the effect of $\mathrm{X}_{\mathrm{c}}(\%)$ on the $\mathrm{T}_{\lambda \text {-directional measurement }}$ varies regarding the wavelength, it opens a discussion about the fact that the microcrystalline structure -in particular spherulites or lamellae substructures- are responsible for optical scattering. Based on these findings; the analyses between the microcrystalline structure and the transmission characteristics of PE were extended performing $\mathrm{T}_{\lambda \text {-directional }}$ and $\mathrm{T}_{\lambda \text {-hemispher- }}$ ical measurements on the third group of PE samples $(0.42,0.90$ and $1.82 \mathrm{~mm}$ thick). Thanks to the analyses, two different transmittance measurements were done on each sample and the information about the scattering characteristics of the PE medium was estimated. Under the assumptions revealed in Section 2, it was estimated that the difference between both
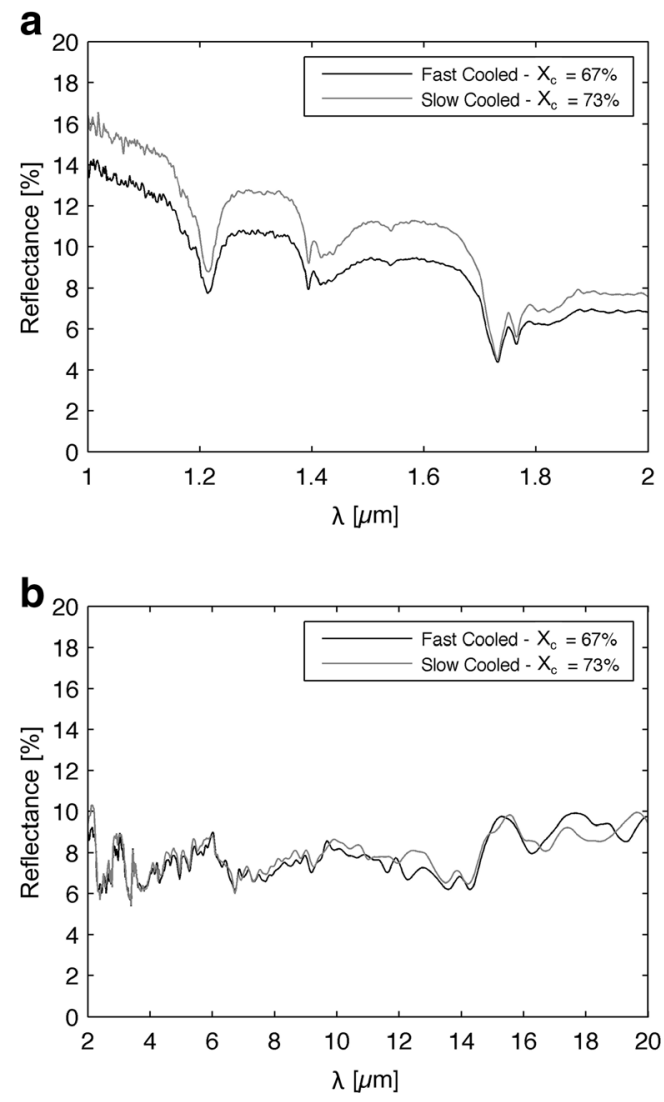

Fig. 5 Reflectance measurements in NIR (a) and MIR ranges (b) on the slow and fast-cooled samples with different $\mathrm{X}_{\mathrm{c}}(\%)$ the measurements represents the amount of the scattered radiation inside of the PE medium. For the sake of clarity of the illustration, only the two samples -the thinnest and thickest ones- are displayed in the Fig. 6. As illustrated in 6 (a), considering the spectral range around $1-1.2 \mu \mathrm{m}$ the difference between the two measurements is around $60 \%$ and $40 \%$ for the 0.42 and $1.82 \mathrm{~mm}$ thick samples. The difference between the estimated scattered radiations obtained from the two samples may be explained by the prolonged optical path of the radiation passing through the medium due to scattering [33] and cause to increase absorption in an equivalent depth, as also reported in [6]. Therefore, the reduction in the estimated scattered radiation may be attributed to the increase in the ratio of the absorbed energy (not in intrinsic absorption) in the thicker sample. Once again, it is crucial for IR-heating radiation models as the maximum emission wavelength of a typical IR lamp is around the corresponding range. It should also be noted that the $\mathrm{T}_{\lambda \text {-directional }}$ measurements on the different samples, that were prepared with identical thickness and thermal treatment (slow or fast cooled), showed that $\mathrm{T}_{\lambda \text {-directional }}$ may vary around $3-5 \%$ in NIR range whereas such a change was not observed for the $T_{\lambda \text {-hemispherical measurements. This is }}$ probably induced by the optical scattering -microcrystalline structure relation which may change by the orientation [12], or position of the sample at each spectroscopic measurement. In
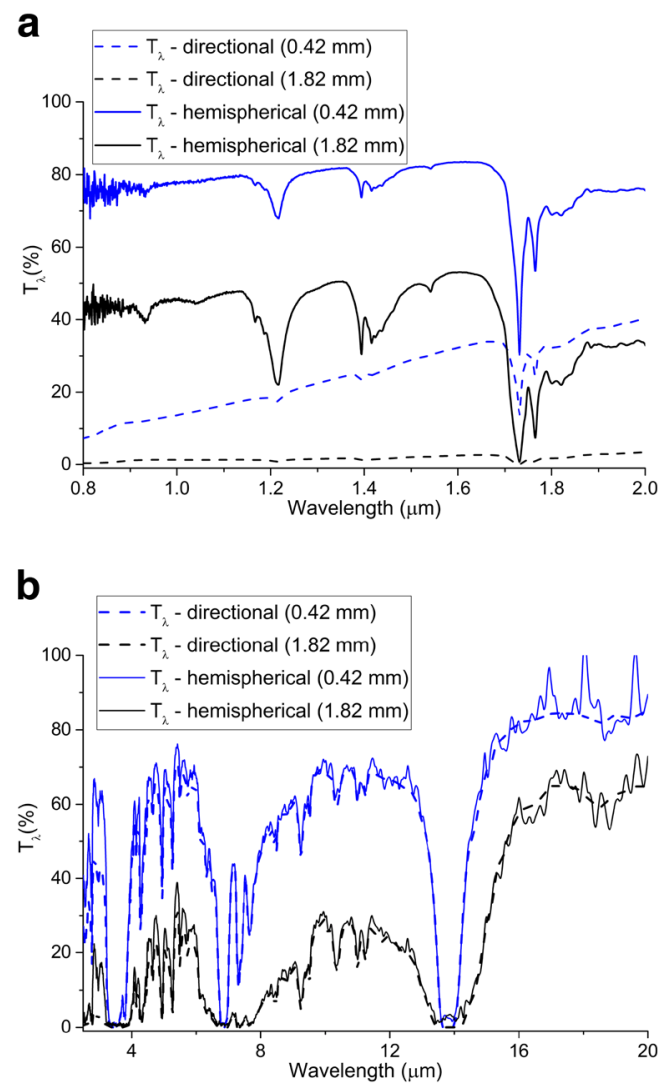

Fig. 6 Comparison between the $T_{\lambda \text {-directional }}$ and $T_{\lambda \text {-hemispherical }}$ measurements of the two identical samples in NIR (a) and MIR ranges (b) 
MIR range, the differences between the measurements on the identical samples are around $20 \%$ for the wavelengths lower than $3.5 \mu \mathrm{m}$ where after the difference gradually decreases and becomes negligibly small. This trend is identical to the first analyses revealed in Fig. 4 (b) that shows the $\mathrm{T}_{\lambda \text {-directional }}$ values of the samples with different $\mathrm{X}_{\mathrm{c}}(\%)$. The same trend from both the analyses are in good agreement with $[12,17]$ where it was concluded that the size of a scatterer in PP semicrystalline polymer is probably too small. Therefore the optical scattering becomes negligible in MIR range due to the increase in wavelength as the size of the scatterers in PP get considerably smaller than the applied wavelength. Based on the findings in the current study and in $[12,17,18]$ it may be concluded that the identification of scatterer is of prime importance to understand the optical scattering phenomenon in semi-crystalline unfilled thermoplastics adequately.

\section{A numerical approach for modeling IR-radiation heat transfer for semi-crystalline polymers}

In order to analyze how the optical scattering affects the temperature predictions in 3D medium, parametric numerical studies were carried out adopting a preliminary numerical approach. In this section, using the optical properties obtained from the two-step experimental analyses, two different thermo-optical properties were proposed which were used as a numerical input for the parametric numerical studies.

In radiation heat transfer problems, radiation propagation is considered in participating medium. The physical meaning of the term "participating medium" is the interaction between a propagated radiation and a medium where the radiation travels into [32]. In other words, the propagated radiation interacts with the medium and it may cause scattering, emission and absorption of radiation that leads generation of heat in the corresponding medium. Considering an initial radiation described as a light that travels alongside a propagation line, it is possible to define the conservation of radiation energy considering all the scattering, absorption and emission phenomena alongside that propagation line. In theory such a definition for the energy balance of radiation energy in participating medium is called as Radiative Transfer Equation (RTE), which is a theoretical backbone of radiative heat transfer problems. The integro - differential form of RTE [23, 32] is stated as in eq. 2:

$$
\begin{aligned}
\frac{d I_{\lambda}}{d S}= & -\beta_{\lambda}(S) I_{\lambda}(S)+\kappa_{\lambda}(S) I_{\lambda b}(S) \\
& +\frac{D_{\lambda}}{4 \pi} \int_{\Omega_{i}=0}^{4 \pi} I_{\lambda}\left(S, \Omega_{i}\right) \varphi_{\lambda}\left(\Omega, \Omega_{i}\right) d \Omega_{i} \text { with; } \beta_{\lambda}=\kappa_{\lambda}+\mathrm{D}_{\lambda}
\end{aligned}
$$

The change in radiative energy after a small path increment (dS) along the radiative propagation direction of $\mathrm{S}$ and solid angle $(\Omega)$ can be calculated based on RTE. The first term in the formula stands for the radiation loss due to both absorption and scattering effects where extinction coefficient $\left(\beta_{\lambda}\right)$ is the sum of both absorption $\left(\kappa_{\lambda}\right)$ and scattering coefficients $\left(D_{\lambda}\right)$. The second term counts the radiation gain due to self-emission of along the $\mathrm{S}$ direction which is function of blackbody intensity $\left(\mathrm{I}_{\lambda b}\right)$. The third term represents the radiation gain caused by in-scattering phenomena where phase function $\left(\varphi_{\lambda}\right)$ defines the change in the direction of propagated radiation due to multiple scattering. If the change in the direction of the propagated radiation is ignored it can then be assumed a homogeneous medium. In addition, considering that the temperature of a heated medium is relatively much lower than the temperature of a heat source, which is an IR-lamp in this case, a cold medium assumption can be adopted. For the current processing conditions, the maximum temperature of a $\mathrm{PE}$ sample and a typical IR-heater will be around $400 \mathrm{~K}$ and $2400 \mathrm{~K}$ respectively and therefore self-emission of the polymer sample can also be neglected. Based on the assumptions of a cold and homogeneous medium for the sample, the second and third terms in RTE are omitted and the radiation intensity at the depth " $\mathrm{S}$ " becomes only the function of attenuated radiation due to extinction, or absorption in case that $\mathrm{D}_{\lambda}=0$. Thus, RTE is reduced and derived to well-known Beer-Lambert Law [2, 3, 5, 6, 34], as shown in Eq. 3:

$\frac{d I_{\lambda}}{d S}=-\beta_{\lambda}(S) I_{\lambda}(S)=-\left(\kappa_{\lambda}(S)+D_{\lambda}(S)\right) I_{\lambda}(S)$

By the same token, the internal heat generation inside of a medium only depends on its extinction characteristics, or its absorption characteristics if $D_{\lambda}=0$. The divergence of radiant flux vector $\left(\mathrm{q}_{\mathrm{r}}\right)$ - which is also called radiative source term - at the depth "S" gives the local heat source due to the absorbed radiation inside of a heated medium (Eq. 4):

$$
\begin{aligned}
\nabla \cdot q_{r}(S) & =\int_{0}^{\infty}-\beta_{\lambda} I_{\lambda}(0) e^{-\beta_{\lambda} S} d \lambda \\
& =\int_{0}^{\infty}-\left(\kappa_{\lambda}+D_{\lambda}\right) I_{\lambda}(0) e^{-\left(\kappa_{\lambda}+D_{\lambda}\right) S} d \lambda
\end{aligned}
$$

Siegel and Howell [23] defined the relation between transmission and reflection characteristics of an optically homogeneous medium in terms of fraction of transmitted light through its medium, which is $T_{\lambda}$, and its intrinsic optical parameters, named as reflectivity $\left(\rho_{\lambda}\right)$ and transmissivity $\left(\tau_{\lambda}\right)$ :

$$
T_{\lambda}=\frac{\left(1-\rho_{\lambda}\right)^{2} \tau_{\lambda}}{1-\left(\rho_{\lambda} \tau_{\lambda}\right)^{2}}
$$

Regarding the optical characteristics of polymers it may be assumed that $\rho_{\lambda}<<1$ and $\rho_{\lambda} \tau_{\lambda}<<1$ [23]. In addition; considering a polymer with a thickness of $h$, the attenuated radiation at the depth $\mathrm{S}=\mathrm{h}$ can be stated as the ratio of the 
transmitted radiation flux to the incident flux. Thus, the radiation attenuation is function of only $\kappa_{\lambda}$ where optical scattering is neglected $\left(D_{\lambda}=0\right)$. Using the $T_{\lambda \text {-directional }}$ measurements, $\kappa_{\lambda}$ was calculated based on Eq. 6 and the radiative source term $\left(\nabla . q_{r}\right)$ was computed using Eq. 4 where a purely absorbing, non-scattering medium was considered.

$T_{\lambda \text {-directional }}=\frac{\phi_{\lambda}^{t}}{\phi_{\lambda}^{i}}=\tau_{\lambda}=e^{-\kappa_{\lambda} . S}$ with $; \beta_{\lambda}=\kappa_{\lambda}$

For the second case of the parametric numerical studies, the thermo-optical parameters of the polymer were determined via

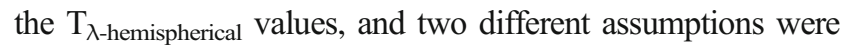
adopted for the polymer medium in NIR and MIR ranges considering the difference between $\mathrm{T}_{\lambda \text {-directional }}$ and $\mathrm{T}_{\lambda \text {-hemispherical }}$ of the identical samples. As aforementioned, the polymer medium shows strong scattering in NIR range where also the material is highly transparent as the $\mathrm{T}_{\lambda \text {-hemispherical }}$ is around $80 \%$. However, the significant difference between $\mathrm{T}_{\lambda \text {-hemispherical }}$ and $\mathrm{T}_{\lambda \text {-directional }}$ in NIR range decreases and vanishes in MIR range indicating that optical scattering becomes negligible. In addition, the transmittance of the tested samples are zero in several spectral bands in MIR range which is known as the absorption bands of PE $[35,36]$ induced by intermolecular interactions and vibrations [37]. Regarding these findings; a homogeneous purely scattering medium with no absorption was assumed [8] in NIR range, whereas only absorption was assumed in MIR range as the optical scattering may be negligible considering all the studied spectral range of MIR, which is between 2 and $20 \mu \mathrm{m}$. Based on these assumptions the relation between $T_{\lambda}$ hemispherical and its thermo-optical properties is defined as:

$$
\begin{gathered}
T_{\lambda \text {-hemispherical }}=\frac{\phi_{\lambda}^{t}+\phi_{\lambda}^{s}}{\phi_{\lambda}^{i}}=e^{-\beta_{\lambda} \cdot d} \text { with; } \beta_{\lambda} \\
=\mathrm{D}_{\lambda} \text { and for } 0.8 \mu \mathrm{m}<\lambda<2 \mu \mathrm{m}
\end{gathered}
$$

$$
T_{\lambda \text {-hemispherical }}=\frac{\phi_{\lambda}^{t}+\phi_{\lambda}^{s}}{\phi_{\lambda}^{i}}=e^{-\kappa_{\lambda} \cdot d} \text { for } 2 \mu \mathrm{m}<\lambda
$$$$
<20 \mu \mathrm{m}
$$

The $\nabla \cdot q_{r}$ stated in Eq. 4 was reformulated in Eq. 9 considering these two spectral ranges:

$$
\begin{aligned}
\nabla \cdot q_{r}(S)= & \int_{0}^{2.2 \mu m}-D_{\lambda} I_{\lambda}(0) e^{-D_{\lambda} S} d \lambda \\
& +\int_{2.2 \mu m}^{20 \mu m}-\left(\kappa_{\lambda}\right) I_{\lambda}(0) e^{-\kappa_{\lambda} S} d \lambda
\end{aligned}
$$

The calculated $\beta_{\lambda}$ and $\kappa_{\lambda}$ over the wavelength between 0.6-15 $\mu \mathrm{m}$ are displayed in Fig. 7. The difference between both the calculated values is significant especially in NIR range. As presented in the fig. $7, \kappa_{\lambda}$ shows much higher values that attributes stronger attenuation of radiation which was calculated using the relatively lower level of transmitted radiative flux in $\mathrm{T}_{\lambda \text {-directional }}$ measurements. However, as displayed in Fig. 6 (a), the amount of flux which was transmitted in $T_{\lambda}$ hemispherical measurements is much higher than the $T_{\lambda \text {-directional }}$ ones indicating that the amount of radiative flux that could not be detected in $\mathrm{T}_{\lambda \text {-directional }}$ measurements was indeed scattered and transmitted but not absorbed.

For the numerical studies, a two-step computation was employed where $\nabla . \mathrm{q}_{\mathrm{r}}$ is computed using an in-house developed radiation heat transfer algorithm - RAYHEAT - and the $\nabla . \mathrm{q}_{\mathrm{r}}$ is used as heat source input in the commercial Finite Element Analysis (FEA) software -COMSOL Multiphysicsto compute the temperature in a 3D geometry. RAYHEAT is a MATLAB-based code which simulates radiation heat transfer using geometrical optics that is based on ray tracing method. Therefore, spectral and direction dependency of the emitted radiation from an IR-lamp are taken into account. Although there are other approaches for modelling radiation transfer, it was demonstrated by Jensen et al. [38] that ray tracing is one of the most accurate method in comparison to the other approaches revealed in literature. In RAYHEAT, the change in the direction of a propagated radiation is only assumed at the surface of a heated medium. Therefore, radiation attenuation in a medium is modeled adopting Beer-Lambert Law as stated in Eq. 4. The accuracy of the algorithm was demonstrated in $[5,39]$ considering a non-scattering $\left(D_{\lambda}=0\right)$, optically homogeneous and cold medium for low-crystalline PET. Detailed information on the computation algorithm and the theoretical background of the model can be found in [5].

As aforementioned, the parametric numerical studies were carried out building two different simulations with the identical boundary conditions, thermal properties and geometrical inputs but with different thermo-optical properties, $\beta_{\lambda}$ and $\kappa_{\lambda}$. The simulations were performed considering a single IR-lamp with a power of $1 \mathrm{~kW}$ and a hollow cylinder made of PE polymer with an internal diameter of $19.7 \mathrm{~mm}$. The thickness

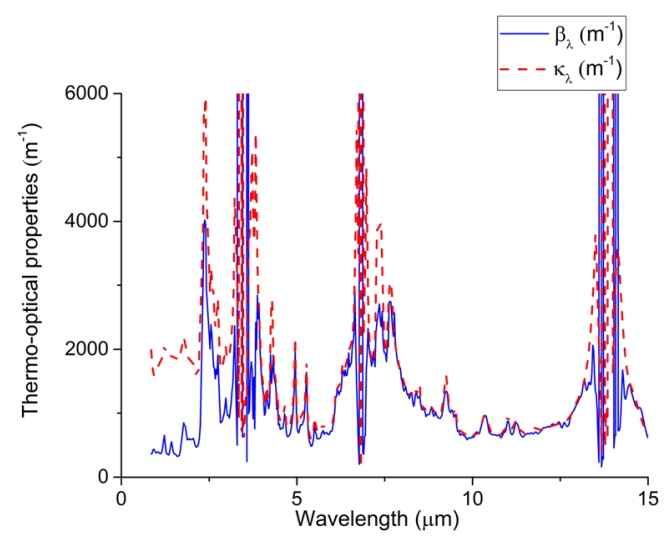

Fig. 7 Spectral $\beta_{\lambda}$ and $\kappa_{\lambda}$ over the range of $0.6-15 \mu \mathrm{m}$ 
of the polymer was defined as $3.3 \mathrm{~mm}$ which was assumed uniform. In RAYHEAT, the models were meshed using linear hexagonal elements and the same mesh was transferred into COMSOL for the second step computations. The hollow cylinder model that represents the PE sample were meshed using first-order hexagonal elements. The temperature of the modeled IR-lamp was set $2400 \mathrm{~K}$ and the radiation emitted from the IR-lamp was modeled using $1.5 \times 10^{6}$ rays. The refractive index of PE was chosen 1.7 [40] and a nonparticipating medium is assumed between the IR-lamp and the heated sample. The geometrical configuration of the simulations is illustrated further in Fig. 8.

$\nabla . q_{\mathrm{r}}$ was computed for each modeled element of the hollow-cylinder geometry, and transferred into COMSOL. A transient heat transfer model was then built for the case of heating the PE sample for $15 \mathrm{~s}$. In other words, the computed $\nabla . \mathrm{q}_{\mathrm{r}}$ for each corresponding volume element were applied for $15 \mathrm{~s}$ to heat the sample up and to predict its final temperature profile at the end of this heating stage. The transient heat transfer equilibrium coupled with the radiation, in terms of $\nabla . \mathrm{q}_{\mathrm{r}}$, can be stated as:

$\rho \cdot C_{p} \frac{\partial T}{\partial t}=\nabla \cdot(k \nabla T)-\nabla \cdot q_{r}$

where $\rho, C_{p}$ and $k$ represent the density, specific heat and thermal conductivity of $\mathrm{PE}$ respectively. Considering the temperature range between 25 and $100{ }^{\circ} \mathrm{C}$, $\rho$ of the material was assumed unchanged under heating and a constant value was adopted from the material data of the manufacturer. The $C_{p}$ was determined using DSC and, a temperature-dependent $C_{p}$ was used as a numerical input in the model. Similarly, variation in thermal conductivity was assumed in the corresponding temperature range and the temperature-dependent $k$ was adopted from literature [10]. These input thermal-physical properties are illustrated in Fig. 9.

In addition, identical boundary conditions were also applied for the transient heat transfer simulations. Natural convection in still air was assumed for the external side of the cylinder.

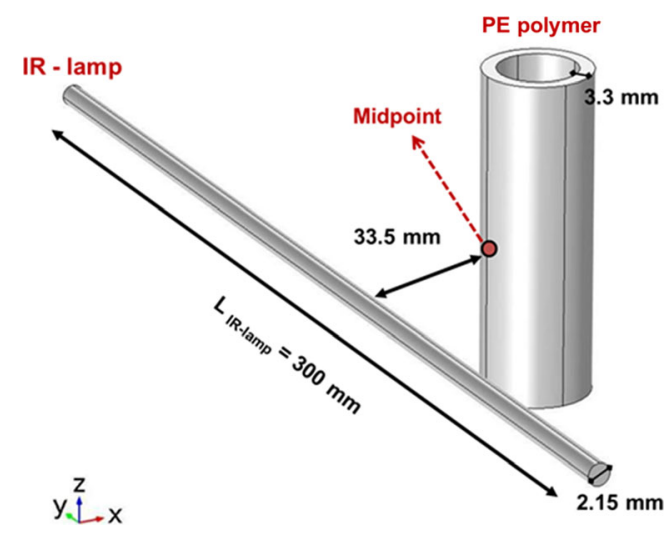

Fig. 8 Schematical representation of the IR-heating simulations of the PE sample used for the parametric numerical studies

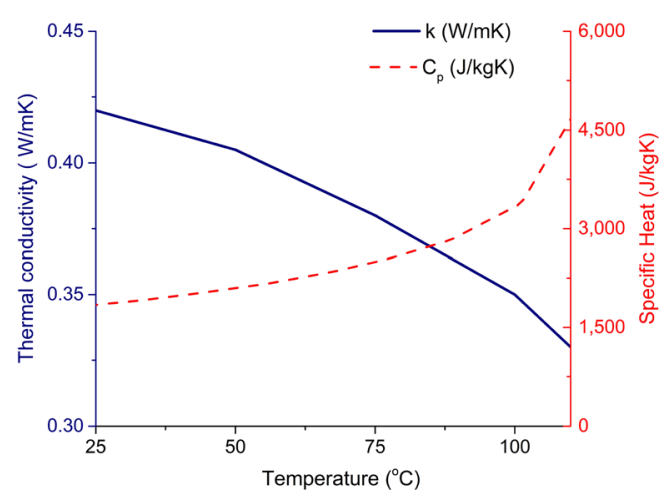

Fig. 9 The temperature-dependent ' $\mathrm{C}_{\mathrm{p}}$ ' and ' $\mathrm{k}$ ' that are measured via DSC analyses and adopted from [10] respectively

Considering natural convection on a vertical surface, the mean convection heat transfer coefficient was chosen as $4.5 \mathrm{~W} / \mathrm{m}^{2} \mathrm{~K}$ [41] and assumed constant throughout the surface. Regarding the cold medium assumption for the PE polymer, self-emission of radiation of the polymer was ignored for both the computations. At the end of the heating stage with $15 \mathrm{~s}$, the throughthickness temperature profiles on the hollow cylinder model were obtained from the two computations and the resulting profiles are compared in Fig. 10. In the figure, " 0 " on the $\mathrm{x}$ axis represents the middle point on the surface of the hollow cylinder that is the closest point to the IR-lamp (displayed in Fig. 8). As it is seen in Fig. 10, the computation-1 that was performed using $\beta_{\lambda}$ shows a relatively lower temperature values where the through-thickness $(\Delta \mathrm{x})$ temperature difference is also smaller than the computation- 2 as a result of weaker radiation attenuation in the medium. The computation- 2 resulted higher absorption of radiation therefore higher temperature on the heated surface of the geometry. In addition, a greater through-thickness temperature difference was computed as a result of stronger radiation attenuation in the medium. However, as it is explained after Figs. 6 and 7, this computation is misleading due to the overestimated absorption characteristics of the PE medium that was calculated using the $\mathrm{T}_{\lambda \text {-directional }}$ measurements.

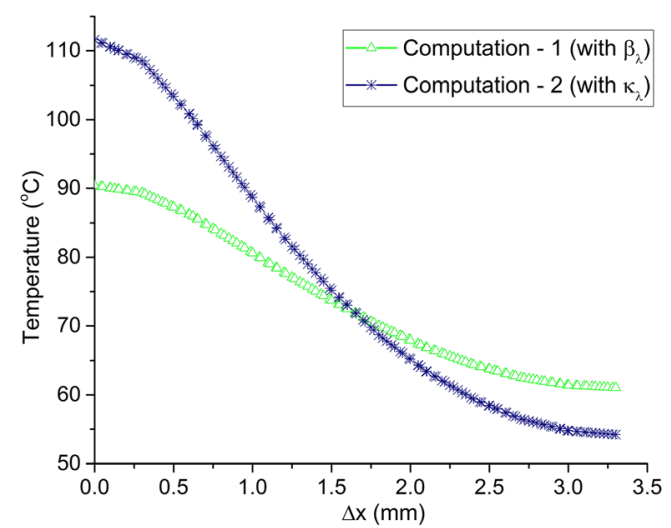

Fig. 10 The through-thickness temperature profiles of the polymer obtained from the two computations performed using the two different thermo-optical properties 
Although 3D temperature profile may not be predicted closely in the highly crystalline PE polymer medium due to the simplifications adopted at this step the parametric numerical analyses demonstrate how the optical scattering may cause to change the predicted temperature, even for a heating case with a single IR-lamp. Considering the fact that the IRheating step of various thermoforming processes of semicrystalline polymers is performed using multiple heat source, the difference between the real and the estimated temperature profile may be much more significant.

\section{Conclusions}

In the current study, the effect of the microcrystalline structure on the thermo-optical properties of PE was experimentally analyzed. The analyses demonstrated that the effect of crystallinity on the transmission was significant considering NIR range, whereas a slight effect was observed in MIR range. Even an increase of 5\% of the $\mathrm{X}_{\mathrm{c}}(\%)$ caused a decrease of nearly $50 \%$ of the $\mathrm{T}_{\lambda \text {-directional }}$ in the spectral range around $1 \mu \mathrm{m}$. Considering IR-heating assisted thermoforming processes, it is crucial to take into account this coupled effect as the maximum emission wavelength of a typical IR heater is close to this spectral range. In addition, the crystallinity does not only affect the transmission through the material but also causes a change in the reflection characteristics as it was also determined that a slight change in crystallinity is somewhat effective on the reflectance levels in NIR range. As the change in the crystalline structure has much greater impact in NIR range than MIR range, the relation between the wavelength of the transmitted light and the size of spherulite or crystalline lamellae may have a key role to change the scattering behavior and therefore the thermo-optical parameters. In other words, the wavelength-dependent effect of crystallinity on the transmittance opens a discussion about the fact that the relation between scatterer size and the wavelength play the key role to affect optical characteristics, particularly transmission due to the optical heterogeneity and scattering of the light. It may therefore be concluded that the change in the thermo-optical characteristics of the PE samples may not be addressed by the $\mathrm{X}_{\mathrm{c}}(\%)$ itself but its microcrystalline morphology, which may defined by the spherulites or their substructures such as lamallae.

The experimental analyses on the transmission characteristics of the polymer were extended adopting comparative experimental measurements on the transmission behavior of the polymer. $T_{\lambda \text {-directional }}$ and $T_{\lambda \text {-hemispherical }}$ of the identical samples were determined and, the amount of the scattered radiative flux was estimated. These measurements also allowed estimating the $\beta_{\lambda}$ and $k_{\lambda}$ of the polymer that were calculated based on a wellknown relation in radiation heat transfer theory. At last, two different models were simulated adopting an identical boundary conditions and numerical inputs but, with two different thermo- optical properties obtained through the comparative experimental analyses. The parametric numerical studies were carried out adopting a preliminary model based on an in-house developed radiation heat transfer algorithm, named as RAYHEAT. Thanks to the numerical analyses the effect of optical scattering, as a numerical input, was analyzed regarding to the predicted through-thickness temperature profiles. Although the analyses are not indicative of the accuracy of the absorbed radiation energy in the polymer medium, and therefore the predicted temperature distributions, it highlights how temperature predictions may differ if the optical scattering phenomenon in semicrystalline thermoplastics is ignored. The $T_{\lambda \text {-hemispherical }}$ measurements clearly revealed that the amount of radiative flux that could not be detected in the $\mathrm{T}_{\lambda \text {-directional }}$ measurements are not absorbed by the polymer medium, but scattered and transmitted. It may therefore be concluded that the computation-1 performed using the $\beta_{\lambda}$ provides relatively more realistic temperature predictions. As a consequence, the absorption characteristics obtained via $T_{\lambda \text {-directional }}$ measurements may require a special attention if an optically heterogeneous medium is considered. Otherwise the thermo-optical properties obtained from such measurements may cause to adopt an erroneous numerical input where temperature predictions may be overestimated due to the incapability of detecting the scattered and transmitted amount of radiative flux. In addition, it is required to model the change in the direction of the scattered radiation in such optically heterogeneous polymer medium for accurate absorbed radiation predictions. In future work, a predictive model will be built including the change in radiation direction induced by optical scattering. The coupled relation between microcrystalline structure and optical characteristics of the polymer will be studied further in order to model optical scattering as a function of microcrystalline structure of the semi-crystalline polymer.

Acknowledgements The authors declare that they have no conflict of interest.

\section{References}

1. Burkhardt G, Hüsgen U, Kalwa M, Pötsch G, Schwenzer C (2011) Plastics processing, 1. Processing of thermoplastics. In: Elvers B (ed) Ullmann's encyclopedia of industrial chemistry. Wiley-VCH Verlag GmbH \& Co. KGaA, Germany, pp 367-404

2. Becker F, Potente H (2002) A step towards understanding the heating phase of laser transmission welding in polymers. Polym Eng Sci 42:365-374

3. Schmidt F (2003) Modelling of infrared heating of thermoplastic sheet used in thermoforming process. J Mater Process Technol 143144:225-231. https://doi.org/10.1016/S0924-0136(03)00291-7

4. Monteix S, Schmidt F, Le Maoult Y et al (2001) Experimental study and numerical simulation of preform or sheet exposed to infrared radiative heating. J Mater Process Technol 119:90-97

5. Cosson B, Schmidt F, Le Maoult Y, Bordival M (2011) Infrared heating stage simulation of semi-transparent media (PET) using ray 
tracing method. Int J Mater Form 4:1-10. https://doi.org/10.1007/ s12289-010-0985-8

6. Geiger M, Frick T, Schmidt M (2009) Optical properties of plastics and their role for the modelling of the laser transmission welding process. Prod Eng 3:49-55. https://doi.org/10.1007/s11740-0080141-1

7. Mayboudi LS (2008) Heat Transfer Modelling and Thermal Imaging Experiments in Laser Transmission Welding of Thermoplastics. Ph.D. thesis, Queen's University

8. Asséko ACA, Cosson B, Schmidt F et al (2015) Laser transmission welding of composites-part a: Thermo-physical and optical characterization of materials. Infrared Phys Technol 72:293-299. https:// doi.org/10.1016/j.infrared.2015.02.004

9. Chen M, Zak G, Bates PJ (2011) Effect of carbon black on light transmission in laser welding of thermoplastics. J Mater Process Technol 211:43-47. https://doi.org/10.1016/j.jmatprotec.2010.08. 017

10. Klein R (2011) Chapter 1- material properties of plastics. In: Laser welding of plastics: materials. Processes and Industrial Applications. Wiley-VCH Verlag $\mathrm{GmbH} \& \mathrm{Co}$. KGaA, Weinheim, Germany, pp 3-69

11. Ilie M, Kneip J-C, Matteï S et al (2007) Laser beam scattering effects in non-absorbent inhomogenous polymers. Opt Lasers Eng 45:405-412. https://doi.org/10.1016/j.optlaseng.2006.07.004

12. Hakoume D, Dombrovsky LA, Delaunay D, Rousseau B (2014) Spectroscopic diagnostics of morphological changes arising in thermal processing of polypropylene. Appl Opt 53:2702. https://doi. org/10.1364/AO.53.002702

13. Hohmann M, Devrient M, Klämpfl F et al (2014) Simulation of light propagation within glass fiber filled thermoplastics for laser transmission welding. Phys Procedia 56:1198-1207. https://doi. org/10.1016/j.phpro.2014.08.035

14. Stokes-Griffin CM, Compston P (2015) Optical characterisation and modelling for oblique near-infrared laser heating of carbon fibre reinforced thermoplastic composites. Opt Lasers Eng 72:111. https://doi.org/10.1016/j.optlaseng.2015.03.016

15. Lebaudy P, Saiter JM, Grenet J, Vautier C (1992) Temperature distribution in poly (ethylene terephthalate) plate undergoing heat treatment. Diffusion influence: 1. Theoretical approach. Polymer 33:1887-1892. https://doi.org/10.1016/0032-3861(92)90488-I

16. Denis A, Dargent E, Lebaudy PH et al (1996) Dependence on the spectral scattering coefficient on crystallinity into semicrystalline polyester. J Appl Polym Sci 62:1211-1218. https://doi.org/10. 1002/(SICI)1097-4628(19961121)62:8<1211::AID-APP11>3.0. $\mathrm{CO} ; 2-\mathrm{A}$

17. Hakoume D, Dombrovsky LA, Delaunay D, Rousseau B (2014) Effect of Processing Temperature on Radiative Properties of Polypropylene and Heat Transfer in the Pure and Glassfibre Reinforced Polymer. https://doi.org/10.1615/IHTC15.rad.008207

18. Boztepe S, Thiam A, de Almeida O, Le Maoult Y, Schmidt F (2016) Experimental analysis on the coupled effect between thermo-optical properties and microstructure of semi-crystalline thermoplastics. $p$ 020006. https://doi.org/10.1063/1.4963410

19. Reiter G, Strobl GR (2007) Progress in understanding of polymer crystallization. Springer, Berlin; New York

20. Zalewski EF (1995) Radiometry and photometry. Handb Opt 2:24-21

21. Apetz R, van Bruggen MPB (2003) Transparent alumina: a lightscattering model. J Am Ceram Soc 86:480-486. https://doi.org/10. 1111/j.1151-2916.2003.tb03325.x
22. Peelen JGJ, Metselaar R (1974) Light scattering by pores in polycrystalline materials: transmission properties of alumina. J Appl Phys 45:216-220. https://doi.org/10.1063/1.1662961

23. Howell JR, Menguc MP, Siegel R (2010) Thermal radiation heat transfer, 5th edition, 5 edition. CRC Press, Boca Raton

24. Kessel J (1986) Transmittance measurements in the integrating sphere. Appl Opt 25:2752. https://doi.org/10.1364/AO.25.002752

25. Manara J, Arduini-Schuster M, Hanssen L (2009) Integrating sphere reflectance and transmittance intercomparison measurements for evaluating the accuracies of the achieved results. High Temp-High Press 38:259-276

26. The role of microcrystalline structure on the temperature-dependent thermo-optical properties of semi-crystalline thermoplastics and non-invasive temperature measurements (2017), $25^{\text {eme }}$ Congrès Français de Thermique, Marseille - France

27. Genna S, Leone C, Tagliaferri V (2017) Characterization of laser beam transmission through a high density polyethylene (HDPE) plate. Opt Laser Technol 88:61-67. https://doi.org/10.1016/j. optlastec.2016.08.010

28. Viscarra Rossel RA, Walvoort DJJ, McBratney AB et al (2006) Visible, near infrared, mid infrared or combined diffuse reflectance spectroscopy for simultaneous assessment of various soil properties. Geoderma 131:59-75. https://doi.org/10.1016/j.geoderma. 2005.03.007

29. Sasic S, Ozaki Y (2011) Raman, infrared, and near-infrared chemical imaging. John Wiley \& Sons, Hoboken

30. Wriedt T (2012) Mie theory: a review. In: Hergert W, Wriedt T (eds) Mie theory. Springer Berlin Heidelberg, Berlin, Heidelberg, pp 53-71

31. Heck B, Kawai T, Strobl G (2006) Time dependent light attenuation measurements used in studies of the kinetics of polymer crystallization. Polymer 47:5538-5543. https://doi.org/10.1016/j.polymer. 2005.11.098

32. Modest MF (2003) Radiative Heat Transfer, Second Edition, 2 edition. Academic Press, Amsterdam; Boston

33. Boglea AL, Roesner A, Russek UA (2011) Laser beam welding of thermoplastics. In: Poprawe R (ed) Tailored light 2. Springer Berlin Heidelberg, Berlin, Heidelberg, pp 284-307

34. Coelho JMP, Abreu MA, Carvalho Rodrigues F (2004) Methodologies for determining thermoplastic films optical parameters at $10.6 \mu \mathrm{m}$ laser wavelength. Polym Test 23:307-312. https:// doi.org/10.1016/j.polymertesting.2003.07.001

35. Bendada A, Cole K, Lamontagne M, Simard Y (2003) A hollow waveguide infrared thermometer for polymer temperature measurement during injection moulding. J opt. Pure Appl Opt 5:464

36. Gulmine J, Janissek P, Heise H, Akcelrud L (2002) Polyethylene characterization by FTIR. Polym Test 21:557-563. https://doi.org/ 10.1016/S0142-9418(01)00124-6

37. DeWitt DP, Nutter GD (1988) Theory and practice of radiation thermometry. John Wiley \& Sons, Hoboken

38. Jensen K, Ripoll J, Wray A et al (2007) On various modeling approaches to radiative heat transfer in pool fires. Combust Flame 148:263-279. https://doi.org/10.1016/j.combustflame.2006.09.008

39. Bordival M, Schmidt FM, Le Maoult $Y$ et al (2010) A ray tracing method to simulate the infrared heating of semi-transparent thermoplastics. Int J Mater Form 3:809-812. https://doi.org/10.1007/ s12289-010-0893-y

40. Biron M (2012) Thermoplastics and thermoplastic composites. William Andrew Publishing, Norwich

41. Faghri A, Zhang Y, Howell JR (2010) Advanced heat and mass transfer. Global Digital Press, Columbia, USA 